\title{
Software-Defined Networking (SDN) and Network Function Virtualization (NFV) for a Hyperconnected World: Challenges, Applications, and Major Advancements
}

\author{
Abbas Bradai ${ }^{1} \cdot$ Mubashir Husain Rehmani ${ }^{2}$. Israat Haque ${ }^{3}$. \\ Michele Nogueira ${ }^{4}$. Syed Hashim Raza Bukhari ${ }^{5}$
}

Published online: 26 May 2020

(c) Springer Science+Business Media, LLC, part of Springer Nature 2020

In recent years, people have become more connected to the network, sharing information, collaborating, and generating/consuming a huge amount of data. This "hyperconnected world" is driven by the next generation of the Internet, where different networks, such as the traditional Internet, Internet of things (IoT), smart cities, smart grids, and intelligent transportation systems, are federated under the umbrella of one network called $5 \mathrm{G}$. Indeed, the $5 \mathrm{G}$ is gaining momentum as it extends the regular Internet by connecting a diverse range of "things" or physical objects like electronic appliances, cars, thermostats, and other devices. The 5G could provide a broad array of services to the society, including weather monitoring, medical services, transportation and vehicular services, defense applications, and smart cities applications.

Abbas Bradai

Abbas.bradai@univ-poitiers.fr

Mubashir Husain Rehmani

mshrehmani@gmail.com

Israat Haque

israat@dal.ca

Michele Nogueira

michele.nogueira@ufpr.br

Syed Hashim Raza Bukhari

hashimbukhari01@gmail.com

1 Xlim Institute, University of Poitiers, Poitiers, France

2 Department of Computer Science, Cork Institute of Technology, Cork, Ireland

3 Faculty of Computer Science, Dalhousie University, Halifax, Canada

4 Department of Computer Science, Federal University of Paraná, Curitiba, Brazil

5 Department of Electrical and Computer Engineering, COMSATS University Islamabad, Attock Campus, Attock, Pakistan 
In this context, Software-Defined Networking (SDN) and Network Function Virtualization (NFV) technologies could play a central role in addressing these challenges. On the one hand, SDN offers more efficient control of the $5 \mathrm{G}$ network thanks to the separation of the control plane from the data plane and its centralized management approach. On the other hand, NFV and virtualization technologies allow the efficient slicing of the network depending on the applications' requirements and promise to provide the flexibility of dynamically provisioning network functions whenever needed. With such benefits, SDN/NFV is becoming the ultimate tool to manage the $5 \mathrm{G}$ network to handle the high number of connected objects and users and to provide high bandwidth traffic and low latency applications.

For this special issue of the Journal of Network and Systems Management, we received 25 articles submissions. After a rigorous peer-review process, we accepted 10 original and non-published papers.

In "Dynamic VNF Placement to Manage User Traffic Flow in Software-Defined Wireless Networks," Tahira Mahboob, Young Rok Jung and Min Young Chung propose a dynamic VNF migration approach which takes into consideration the user location as well as the available resources at the physical infrastructure nodes. Authors model the problem as ILP and propose a heuristic to solve it. The proposed scheme's performance is evaluated using simulations and an experimental testbed for different scenarios (and under different network topologies). Results show that the proposed solution balances the load on NFV nodes, reduces SFC blocking rates, and improves the network throughput.

In "A GRASP-based Approach for Dynamic Cache Resources Placement in Future Networks", Hamza Ben-Ammar and Yassine Hadjadj-Aoul tackle the problem of placement of a specific VNF, namely the network storage or caching. Authors model the problem as a multi-objective optimization problem, in which both the average distance from which contents are retrieved and the peering links utilization are minimized. The authors propose an adapted version of the Greedy Randomized Adaptive Search Procedure (GRASP) meta-heuristic to solve it. The proposed approach shows its efficiency for different traffic patterns and cache size cases.

In "Toward a machine learning and software-defined network approaches to manage miners' reputation in blockchain", Abdellah Kaci and Abderrezak Rachedi propose an SDN and blockchain-based reputation model called PoolCoin based on a distributed trust model for a mining pool. The proposed scheme's parameters were optimized to exclude only misbehaving miners and to protect mining pools from attacks such as DDoS and BWH attacks.

In "A Holistic Framework for Virtual Network Migration to Enhance Embedding Ratios in Network Virtualization Environments", Mahboobeh Zangiabady, Alberto Garcia-Robledo, Christian Aguilar-Fuster, and Javier Rubio-Loyola propose a virtual network migration framework. This framework considers different parameters, such as CPU capacity, migration policy, and trigger conditions. The proposed framework is compared against two existing schemes and outperformed them in terms of acceptance ratios.

In "Improving switch-to-controller assignment with load balancing in multi-controller software-defined WAN (SD-WAN)", Ali Elkamel and Habib Youssef propose the Distributed Hungarian-based Assignment Protocol (DHAP) to solve the performance issue of a centralized controller-based SDN. As the number of requests increases in 
the central controller environment, the processing may increase the delay and degrade the performance. To optimize the switch-to-controller assignment problem, authors propose their DHAP scheme for a multi-controller context, aiming to achieve a tradeoff between round trip time and controller load. The proposed method shows improvements in flow setup time and load balancing.

In "Applicability of SDN and NFV techniques for a virtualization-based roaming solution", Luis M. Contreras, Luca Cominardi, Jorge Martin Perez, and Carlos J. Bernardos propose the design of a virtualized-based roaming solution using NFV to facilitate the roaming subscribers. The proposed scheme also focuses on the challenges faced by mobile operators due to regulatory issues. Experimental results show the efficiency of the proposed scheme in terms of service creation and activation, as well as reduced latency. The authors also highlight the techno-economic insight for the mobile operators.

In "Application-Aware Firewall Mechanism For Software Defined Networks", Fahad N. Nife and Zbigniew Kotulski follow the promising approach of abstracting network services to propose an interesting application-aware firewall mechanism for software-defined networks. The firewall can be implemented as an extension to the network controller. It can filter network traffic in the application, transport and network level, and identify running applications to enforce security policies. The authors present an interesting discussion of the state-of-the-art related to firewall proposals for SDN and detailed background for the main concepts addressed in the article. The application-aware firewall is implemented in Mininet as a modular application running on top of a Python-based POX SDN controller framework. The authors conclude the article highlighting a set of insightful open issues for future work.

In "Network slicing cost allocation model", Asma Chiha, Marlies Van der Wee, Didier Colle, and Sofie Verbrugge propose a cost allocation model for network slicing. The goal of this work is to allocate the network's cost to the different network slices deployed on the network while fulfilling its KPI requirements, as well as derive how much cost can be saved by using NFV on the core network. The use case considered in this work is based on two slices: IoT and eMBB.

The article "Adaptive Telemetry for Software-Defined Mobile Networks" proposes a new programmable active network telemetry measurement system for $5 \mathrm{G}$ for better scalability and accuracy. The in-switch general-purpose processors enable accurate measurements for a broad set of user-defined tasks. An SDN controller exploits its global network view to coordinate measurement campaigns. The authors define an API based on finite state machines to configure their system and enable operators to set up stateful monitoring tasks. Experimental results show that ATS can provide higher accuracy compared to a controller-based measurement approach.

Service providers usually manually build NFV deployment descriptors to accommodate the topology of network services, network function definitions, and corresponding resource requirements. The manual process is time-consuming, error-prone, and static. "Configurable Deployment Descriptor Model in NFV" designs an automated NFV deployment descriptor generation model to deploy required VNFs dynamically. The evaluation results show the feasibility and utility of the proposed solution. 